\title{
MICRONIZAÇÃO DO AGENTE ATIVO FARMACÊUTICO GLIBENCLAMIDA POR PROCESSO DE PRECIPITAÇÃO COM ANTISSOLVENTE LÍQUIDO
}

\author{
C. S. D. $\operatorname{COSTA}^{1}$ e G. L. V. COELHO ${ }^{1}$ \\ ${ }^{1}$ Universidade Federal Rural do Rio de Janeiro, Departamento de Engenharia Química \\ E-mail para contato: coelho@ufrrj.br
}

\begin{abstract}
RESUMO - A glibenclamida é um potente agente hipoglicemiante oral que tem sido muito utilizado no tratamento do diabetes mellitus não insulino-dependente, porém possui baixa solubilidade aquosa e, consequentemente, baixa biodisponibilidade, podendo constituir uma barreira no desenvolvimento de formas farmacêuticas. Visto que partículas menores possuem maior taxa de dissolução, utilizou-se o arranjo ortogonal $\mathrm{OA}_{16}(4)^{5} \mathrm{com}$ o intuito de otimizar o processo de precipitação com antissolvente líquido e assim produzir partículas de tamanho reduzido, tendo em vista que tal planejamento permite avaliar o efeito de todas as variáveis que podem influenciar o tamanho das partículas. Nas condições ótimas (A4, B2, C3, D3 e E3), sendo a concentração da solução a variável de

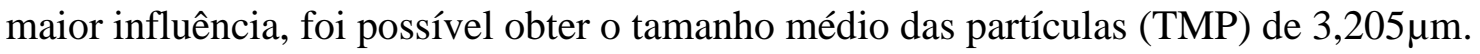

\section{INTRODUÇÃO}

A glibenclamida (GLIB, de fórmula molecular $\mathrm{C}_{23} \mathrm{H}_{28} \mathrm{ClN}_{3} \mathrm{O}_{5} \mathrm{~S}$ e peso molar $494 \mathrm{~g} / \mathrm{mol}$ ) é um potente agente hipoglicemiante oral que tem sido muito utilizado no tratamento do diabetes mellitus não-insulino-dependente, e sua principal ação é estimular a liberação endógena de insulina pelas células beta do pâncreas (ALBU et al., 2007; LI et al., 2013; SHAH et al., 2013).

Para que o medicamento seja absorvido pelo organismo, são necessárias duas etapas: a dissolução seguida pela permeação da mesma na membrana gastrointestinal (SETHIA e SQUILLANTE, 2003; YUKSEL et al., 2000). Diante disso, a solubilidade é um dos principais obstáculos relacionados ao uso da GLIB, já que ela está contida na classe II do Sistema de Classificação Biofarmacêutico, ou seja, a droga possui baixa solubilidade e alta permeabilidade (NETO, 2012; PUIGJANER e PROHENS, 2007; REHDER et al., 2012; SHAH et al., 2013; WEI et al., 2008).

Em drogas de alta permeabilidade, a biodisponibilidade oral é limitada pela taxa de dissolução (MATTEUCCI et al., 2006) e, por este motivo, diversas técnicas tem sido utilizadas com o intuito de melhorar a solubilidade e, consequentemente, a biodisponibilidade oral da substância, como a redução do tamanho das partículas (YU et al., 2011), o uso de surfactantes, formação de dispersões sólidas, transformação de formas cristalinas para o estado amorfo, sendo que a primeira tem se destacado como a opção mais efetiva (ZHANG et al., 2009). 


\section{9 a 22 de outubro de 2014 \\ Florianópolis/SC}

\subsection{Precipitação com Antissolvente Líquido (PAL)}

A micronização por precipitação com antissolvente líquido é uma técnica promissora para a redução do tamanho das partículas quando a substância em questão é moderadamente solúvel em um solvente em particular, sendo que o processo é rápido, de baixo custo e de fácil industrialização. (ZHANG et al., 2009; ZHAO et al., 2013).

Na PAL, diversas variáveis externas, como concentração da solução, intensidade de agitação, razão de volume antissolvente/solvente e tempo de precipitação podem afetar as propriedades dos cristais formados (ZHAO et al., 2013). Além disso, aditivos externos podem ser utilizados para inibir a aglomeração e evitar que grandes cristais sejam formados (KIM et al., 2014).

Neste trabalho, as micropartículas de GLIB foram produzidas por precipitação com antissolvente líquido, tendo como objetivos melhorar a taxa de dissolução e a biodisponibilidade oral da mesma, avaliar os fatores que afetam o tamanho médio das partículas (TMP) e otimizar o processo, sendo que para tal foi utilizando o arranjo ortogonal $\mathrm{OA}_{16}(4)^{5}$. Foram feitas análises de distribuição do tamanho das partículas e testes para verificar a solubilidade da GLIB em alguns solventes orgânicos.

\section{MATERIAIS E MÉTODOS}

\subsection{Materiais}

Glibenclamida (CAS 10238-21-8) foi doada pela Fundação para o Remédio Popular (FURP Guarulhos, SP, Brasil). Etanol (VETEC, 99,8\%) foi usado como solvente orgânico para a GLIB, TWEEN $^{\circledR} 80$ (CAS 9005-65-6) foi adquirido da Sigma-Aldrich e utilizado como aditivo externo, sendo a água destilada utilizada como antissolvente. Metanol (VETEC, 99,8\%), Acetona (VETEC, 99,8\%) e Isopropanol (VETEC, 99,5\%) foram utilizados, juntamente com o etanol, para verificar a solubilidade da GLIB.

\subsection{Determinação da solubilidade}

A solubilidade da GLIB (mg.ml ${ }^{-1}$ ) foi testada nos solventes orgânicos etanol, metanol, acetona e isopropanol. Para tal, cerca de $10 \mathrm{mg}$ da droga foram cuidadosamente pesados e colocados em um béquer, no qual uma alíquota de $1 \mathrm{ml}$ do solvente foi adicionada com o auxílio de uma pipeta até a completa dissolução da droga. Todo o procedimento foi feito sob agitação magnética e repetido no mínimo 3 vezes.

\subsection{Micronização da glibenclamida}

A GLIB micronizada foi preparada através do processo de precipitação com antissolvente líquido, sendo que o diagrama do processo experimental pode ser observado na Figura 1. 


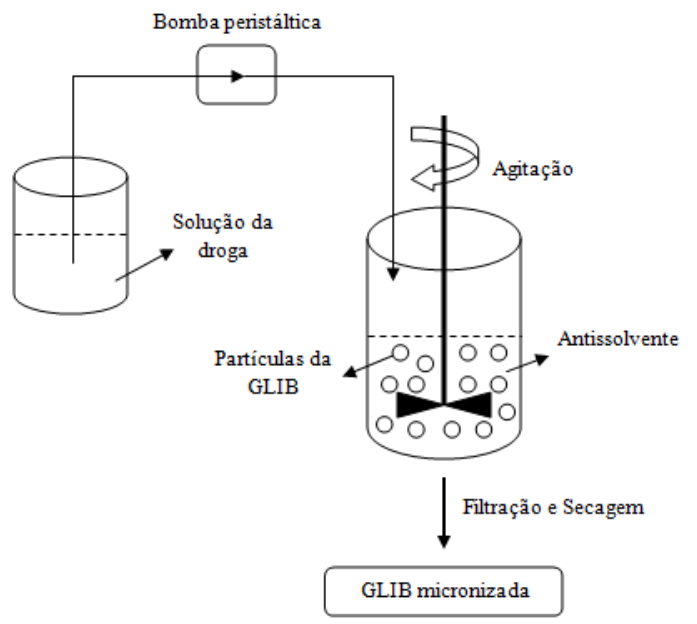

Figura1 - Diagrama do processo experimental.

A GLIB foi dissolvida no solvente orgânico e a solução de concentração previamente definida foi filtrada através de uma membrana Millipore ${ }^{\circledR}$ de $0,22 \mu \mathrm{m}$ para retirar possíveis impurezas. Utilizando uma bomba peristáltica com um fluxo de $15 \mathrm{ml}^{\mathrm{min}}{ }^{-1}$, a solução foi injetada no antissolvente contendo uma quantidade do aditivo externo sob agitação e a precipitação das partículas ocorreu imediatamente. Depois de certo tempo, a suspensão esbranquiçada foi centrifugada a 5000 rpm por cinco minutos, as partículas foram filtradas e secas na Mufla (EDG $3 \mathrm{P}-\mathrm{S} 1800)$ à $70^{\circ} \mathrm{C}$ por 2 horas e estocadas para uso. Todos os experimentos foram realizados a temperatura ambiente.

\subsection{Otimização do processo}

$\mathrm{O}$ arranjo ortogonal $\mathrm{OA}_{16}(4)^{5}$ foi utilizado para otimizar as condições de operação do processo, sendo que permite avaliar o efeito de até cinco fatores com quatro níveis na variável resposta (TMP). Os fatores avaliados foram concentração da solução (A), razão de volume antissolvente/solvente (B), intensidade de agitação (C), tempo de precipitação (D) e concentração de TWEEN ${ }^{\circledR} 80$ (E), estando estes dispostos na Tabela 1, juntamente com os respectivos níveis. Os níveis de cada fator foram baseados nos resultados de experimentos preliminares, sendo que A variou de 0,5-2,0 mg. $\mathrm{ml}^{-1}$, B de 3-8, C de 300-1550 rpm, D de 3-12 min e E de 0-0,3 (\% v/v). Os 16 experimentos foram gerados pelo Statistica ${ }^{\circledR} 10$ (USA). 


\section{9 a 22 de outubro de 2014 \\ Florianópolis/SC}

Tabela 1 - Fatores e níveis do $\mathrm{OA}_{16}(4)^{5}$

\begin{tabular}{cccccc}
\hline Nível/fator & $\mathrm{A}\left[\mathrm{mg} \cdot \mathrm{ml}^{-1}\right]$ & $\mathrm{B}$ & $\mathrm{C}[\mathrm{rpm}]$ & $\mathrm{D}[\mathrm{min}]$ & $\mathrm{E}[\% \mathrm{v} / \mathrm{v}]$ \\
\hline 1 & 0,5 & 3 & 300 & 3 & 0 \\
2 & 1,0 & 4 & 660 & 5 & 0,1 \\
2 & 1,5 & 6 & 990 & 10 & 0,2 \\
4 & 2,0 & 8 & 1550 & 12 & 0,3 \\
\hline
\end{tabular}

Após definida a condição ótima do processo, as micropartículas foram preparadas e analisadas.

\subsection{Caracterização}

Tamanho médio e distribuição do tamanho das partículas: o TMP e a distribuição do tamanho das partículas foi determinado usando o Mastersizer 2000 (Malvern), sendo utilizada uma bomba com velocidade de $3300 \mathrm{rpm}$ e ultrassom.

\section{RESULTADOS E DISCUSSÃO}

\subsection{Determinação da solubilidade}

A micronização por precipitação com antissolvente é utilizada quando uma substância é moderadamente solúvel em um determinado solvente. Sendo assim, a escolha deste é de grande importância para o sucesso do processo. A solubilidade da GLIB em etanol, metanol, acetona e isopropanol é 2,01, 2,20, 5,02 e 0,998 mg.ml ${ }^{-1}$, respectivamente. O isopropanol apresenta a menor solubilidade e, devido a isso, não foi escolhido. A toxicidade dos solventes deve ser baixa o suficiente para não prejudicar a saúde. Portanto, os solventes orgânicos são distribuídos em três classes (1, 2 e 3) de acordo com o seu nível de toxicidade, sendo da classe 3 os menos tóxicos. Logo, por estar contido na classe 2, o metanol também foi descartado. Etanol e acetona são solventes da classe 3, mas o primeiro foi escolhido por ser um composto de baixo ponto de ebulição, baixo custo e de fácil obtenção.

\subsection{Otimização}

Todos os fatores foram examinados usando o arranjo ortogonal $\mathrm{OA}_{16}(4)^{5}$ e o TMP das partículas estão listados na Tabela 2. 
Tabela 2 - Combinações do $\mathrm{OA}_{16}(4)^{5}$ e o TMP resultante.

\begin{tabular}{|c|c|c|c|c|c|c|}
\hline Exp. & $\mathrm{A}\left[\mathrm{mg} \cdot \mathrm{ml}^{-1}\right]$ & $\mathrm{B}$ & $\mathrm{C}$ [rpm] & $\mathrm{D}$ [min] & $\mathrm{E}(\% \mathrm{v} / \mathrm{v})$ & $\mathrm{TMP}[\mu \mathrm{m}] \pm \mathrm{DP}$ \\
\hline 1 & 0,5 & 3 & 300 & 3 & 0 & $92,117 \pm 1,9723$ \\
\hline 2 & 0,5 & 4 & 660 & 5 & 0,1 & $13,376 \pm 0,1469$ \\
\hline 3 & 0,5 & 6 & 990 & 10 & 0,2 & $11,877 \pm 0,1717$ \\
\hline 4 & 0,5 & 8 & 1550 & 12 & 0,3 & $15,784 \pm 0,2300$ \\
\hline 5 & 1,0 & 3 & 660 & 10 & 0,3 & $11,443 \pm 0,1896$ \\
\hline 6 & 1,0 & 4 & 300 & 12 & 0,2 & $12,354 \pm 0,3058$ \\
\hline 7 & 1,0 & 6 & 1550 & 3 & 0,1 & $6,212 \pm 0,2660$ \\
\hline 8 & 1,0 & 8 & 990 & 5 & 0 & $6,320 \pm 0,0995$ \\
\hline 9 & 1,5 & 3 & 990 & 12 & 0,1 & $8,251 \pm 0,0278$ \\
\hline 10 & 1,5 & 4 & 1550 & 10 & 0 & $5,722 \pm 0,2423$ \\
\hline 11 & 1,5 & 6 & 300 & 5 & 0,3 & $13,963 \pm 0,4102$ \\
\hline 12 & 1,5 & 8 & 660 & 3 & 0,2 & $6,600 \pm 0,3328$ \\
\hline 13 & 2,0 & 3 & 1550 & 5 & 0,2 & $5,642 \pm 0,1211$ \\
\hline 14 & 2,0 & 4 & 990 & 3 & 0,3 & $5,715 \pm 0,0380$ \\
\hline 15 & 2,0 & 6 & 660 & 12 & 0 & $7,373 \pm 0,3958$ \\
\hline 16 & 2,0 & 8 & 300 & 10 & 0,1 & $10,090 \pm 0,0831$ \\
\hline $\mathrm{M}_{1}$ & 33,249 & 29,364 & 32,131 & 27,661 & 27,883 & \\
\hline $\mathrm{M}_{2}$ & 9,082 & 9,292 & 9,698 & 9,825 & 9,482 & \\
\hline $\mathrm{M}_{3}$ & 8,634 & 9,817 & 8,001 & 9,744 & 9,079 & \\
\hline $\mathrm{M}_{4}$ & 7,205 & 9,699 & 8,340 & 10,941 & 11,726 & \\
\hline D & 26,044 & 20,072 & 24,130 & 17,917 & 18,804 & \\
\hline Nível ótimo & A3 & B2 & $\mathrm{C} 3$ & D3 & E3 & \\
\hline
\end{tabular}

$\mathrm{M}_{1}=\Sigma\left(\right.$ tamanho médio das partículas em $\left.\mathrm{A}_{1}\right) / 4$.

$\mathrm{D}=\mathrm{Mi}_{\text {máx }}-\mathrm{Mi}_{\text {mín }}$ 
A Tabela 2 indica que o maior TMP foi $92,117 \mu \mathrm{m}$ e o menor foi $5,642 \mu \mathrm{m}$, sendo estes obtidos nos experimentos 1 e 13, respectivamente. De acordo com o valor $\mathrm{D}$, fica claro que a influência dos fatores, em ordem decrescente, é: A > B > C > E > D. Então, o menor TMP é obtido em A4, B2, C3, D3 e E3. Diante disso, foi feito um experimento confirmatório no qual o TMP obtido foi 3,205 $\mu \mathrm{m}$. Nota-se que houve uma redução no TMP, sendo que esta pode ser melhor entendida quando se analisa a distribuição do tamanho das partículas: TMP 5,642 $\mu \mathrm{m}[\mathrm{d}(0,1)=1,926 ; \mathrm{d}(0,5)=4,696 ; \mathrm{d}(0,9)=$ $10,791]$ e TMP $3,205 \mu \mathrm{m}[\mathrm{d}(0,1)=1,417 ; \mathrm{d}(0,5)=2,838 ; \mathrm{d}(0,9)=5,395]$. Observa-se que no experimento final $90 \%$ das partículas estão abaixo de $5,395 \mu \mathrm{m}$, fazendo com o TMP seja menor (Figura 2).

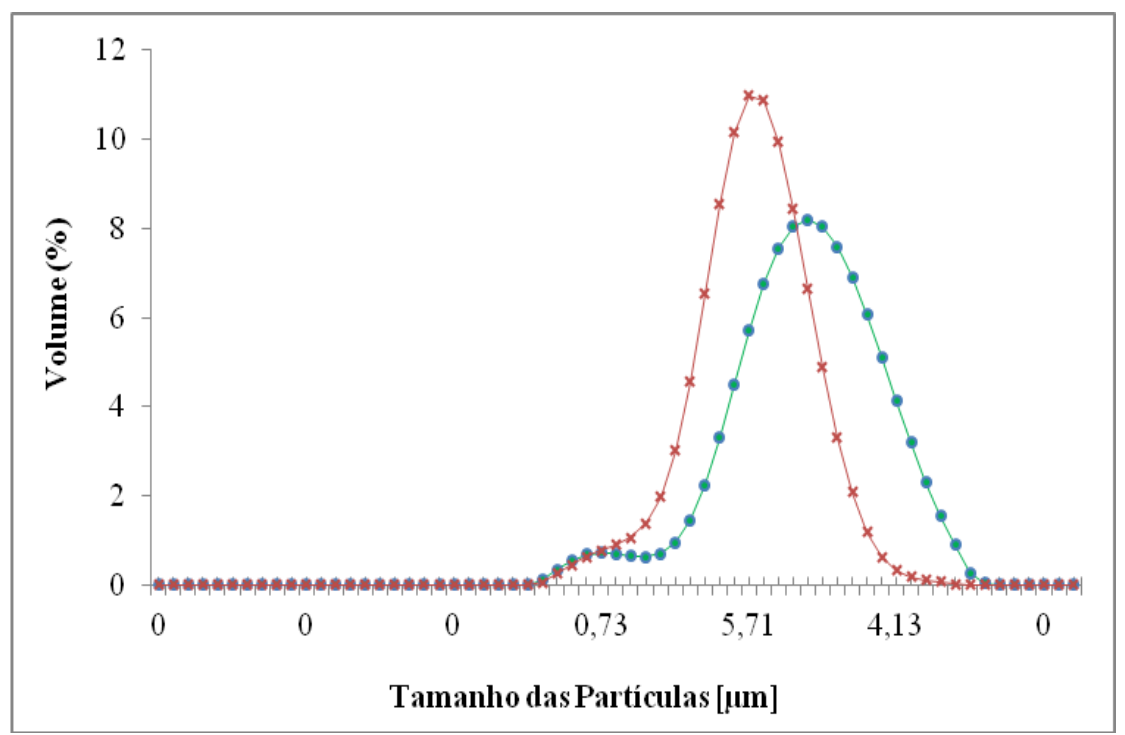

Figura 2 - Distribuição do tamanho das partículas: (• ) GLIB obtida no experimento 13; ( * GLIB obtida no experimento final.

Tendo em vista que os parâmetros experimentais podem afetar diretamente o TMP, a otimização do processo é um ponto crítico para o desenvolvimento da PAL.

\section{CONCLUSÕES}

A precipitação com antissolvente líquido foi utilizada para produzir micropartículas de glibenclamida, a fim de se melhorar sua solubilidade, taxa de dissolução e, consequentemente, a biodisponibilidade oral. O etanol foi utilizado como solvente, a água como antissolvente, concentração da solução [mg.ml ${ }^{-1}$ ], razão de volume antissolvente/solvente, intensidade de agitação [rpm], tempo de precipitação [min] e concentração de aditivo [\%v/v] foram as variáveis avaliadas e TMP $[\mu \mathrm{m}]$ a variável dependente, sendo a concentração da solução o fator de maior influência no TMP e o ponto ótimo obtido em A3, B2, C3, D3 e E3. Neste, foram obtidas partículas com uma distribuição de tamanhos mais estreita e um TMP de 3,205 $\mu \mathrm{m}$. Entretanto, características como morfologia, estrutura química, cristalinidade, estabilidade térmica e solvente residual devem ser realizadas para garantir que o insumo farmacêutico seja seguro para consumo. 


\section{REFERÊNCIAS}

ALBU, F.; GEORGITA, C.; DAVID, V.; MEDVEDOVICI, A. Determination of glibenclamida in human plasma by liquid chromatography and atmospheric pressure chemical ionization/MS-MS detection. J. of Chromat. B, 846, p. 222-229, 2007.

LI, N.; DENG, Y.; WANG, D.; QIAO, Y.; LI, F. Determination of glibenclamide and puerarin in rat plasma by UPLC-MS/MS: Application to their pharmacokinetic interaction study. Talanta, 104, p. 109-115, 2013

SHAH, S. R.; PARIKH, R. H.; CHAVDA, J. R.; SHETH, N. R. Application of PlackettBurman screening design for preparing glibenclamide nanoparticles for dissolution enhancement. Powder Tec., 235, p. 405-411, 2013.

SETHIA, S.; SQUILLANTE, E. Solid Dispersions: Revival with Greater Possibilities and Applications in Oral Drug Delivery. Crit. Ver. ${ }^{\text {TM }}$ in Therap. Drug Carrier Syst., 20, p. 215-247, 2003.

YUKSEL, N.; KANIK, A. E.; BAYKARA, T. Comparison of in vitro dissolution profiles by ANOVA-based, model-dependent and-independent methods. Intern. J. of Pharm., 209, p. 5767,2000

NETO, S. A. de L. Preparação e caracterização analítica de dispersões sólidas cristalinas de glibenclamida, obtidas através de secagem por aspersão. 2012. 102 p. Tese (Doutor em Produtos Naturais e Sintéticos Bioativos) - Centro de Ciências da Saúde, Universidade Federal da Paraíba, João Pessoa, 2012.

PUIGJANER, C.; PROHENS, R. Polimorfismo en la industria farmacêutica. El Farmac., n. 373, p. 58-68, 2007.

REHDER, S.; SAKMANN, A; RADES, T.; LEOPOLD, C. S. Thermal degradation of amorphous glibenclamide. Eur. J. of Pharm. and Biopharm., 80, p. 203-208, 2012.

WEI, H.; DALTON, C.; MASO, M. D.; KANFER, I.; LÖNBERG, R. Physicochemical characterization of five glyburide powders: A BCS based approach to predict oral absorption. Eur. $J$. of Pharm. and Biopharm., 69, p. 1046-1056, 2008.

MATTEUCCI, M. E.; HOTZE, M. A.; JOHNSTON, K. P.; WILLIAMS III, R. O. Drug Nanoparticles by Antisolvent Precipitation: Mixing Energy versus Surfactant Stabilization. Langmuir, v. 22, p. 8951-8959, 2006.

YU, L.; LI, C.; LE, Y.; CHEN, J.; ZOU, H. Stabilized amorphous glibenclamide nanoparticles by high-gravity technique. Mat. Chem. and Phys., 130, p. 361-366, 2011. 
ZHANG, H.; WANG, J.; ZHANG, Z.; LE, Y.; SHEN, Z.; CHEN, J. Micronization of atorvastatin calcium by antisolvent precipitation process. Int. J. of Pharm., 374, p. 106-113, 2009.

ZHAO, X.; SONG, K.; WANG, S.; ZU, Y.; LI, N.; YU, X. Micronization of the Pharmaceutically Active Agent Genipin by an Antisolvent Precipitation Process. Chem. Eng. Tech., v. 36, n. 1, p. 33-42, 2013.

KIM, S.; KIM, H.; YEO, S. Crystallization of silibinin from organic solutions using supercritical and aqueous antisolvents. J. of Super. F., 85, p. 102-109, 2014. 